\title{
Inducing blindsight in normal observers
}

\author{
LEONARD ROBICHAUD \\ Carleton University, Ottawa, Ontario, Canada \\ and \\ LEW B. STELMACH \\ Carleton University and Communications Research Centre, Ottawa, Ontario, Canada
}

\begin{abstract}
We attempted to induce blindsight in normal observers, in an effort to replicate and extend the findings of Kolb and Braun (1995). In that demonstration, observers were able to localize a target in the absence of visual awareness, indicated by the lack of a correlation between localization accuracy and confidence ratings. Replication of this work seemed essential, given the failed attempt by Morgan, Mason, and Solomon (1997). A key aspect of the present work was the use of a pointing response, which is believed to have access to the unconscious representations subserving blindsight. In the critical rivalrous condition, the display consisted of Gabor patterns presented dichoptically with orthogonal orientation in each eye. Binocular summation of left and right images combined to give the appearance of a uniform plaid, camouflaging the texture-defined target. Our attempt to demonstrate blindsight in normal observers was unsuccessful, in that the localization accuracy of the texture-defined target and the observers' confidence ratings were positively correlated. Although the replication was unsuccessful, the results are valuable in that they provide closure to this widely publicized but fruitless line of inquiry.
\end{abstract}

Can blindsight ${ }^{1}$ be induced in normal observers? Kolb and Braun (1995) provided an affirmative answer to this question in a study employing an innovative texturesegmentation task, in which observers were required to localize a texture-defined target in a stimulus complex such as that shown in Figure 1. In the critical rivalrous condition, the Gabor micropatterns had a complementary orientation in each eye, and, when fused, took on the appearance of a uniform plaid. In Kolb and Braun's report, blindsight was manifested in the rivalrous condition: Participants were unaware of seeing the target, but were able to localize it accurately; confidence ratings were uncorrelated with the accuracy of their localization responses. By comparison, in the nonrivalrous condition, in which the Gabor micropatterns had the same orientation in each eye, observers were both aware of seeing the target and able to localize it, and confidence ratings were correlated with the accuracy of their localization responses.

Although the observers in Kolb and Braun's (1995) experiment did not have a lesion and, thus, could not exhibit clinical blindsight, it was argued that the rivalrous display had perceptual effects analogous to those of a lesion. It impeded the direct propagation of information from striate cortex to cortical areas responsible for visual awareness, but it did not impede the transmission of information about target location to cortical areas responsible for visuomotor performance. A path to the latter areas

Correspondence concerning this article may be addressed to L. Stelmach, Communications Research Centre, 3701 Carling Ave., Ottawa, ON, K2H 8S2, Canada (e-mail: lew.stelmach@crc.ca). via the superior colliculus or pulvinar nucleus is believed to subserve residual visuomotor function in patients (Milner \& Goodale, 1995, p. 40) and could explain the dissociation between localization accuracy and confidence ratings in normal observers.

Given the important implications of Kolb and Braun's (1995) results for understanding visual perception and action, our goal was to replicate and extend their work. Replication of their study seemed essential, because of the paradigm-breaking status of the work (see the editorial by Georgeson, 1997) and because of the failed replication by Morgan, Mason, and Solomon (1997). We repeated the original experiment and added a new condition, which employed a pointing response. Following others (Milner \& Goodale, 1995, p. 78; Perenin \& Jeannerod, 1978), we reasoned that a pointing response is a more direct measure of visuomotor ability than is the original verbal-response procedure, and, therefore, would be more likely to reveal blindsight. In comparison with the verbal response, the pointing response would depend more directly on activity in the dorsal stream of the brain - that is, the stream responsible for visuomotor guidance (Milner \& Goodale, 1995). A pointing response could potentially be more accurate in localizing the target in those situations in which observers were unable to report its location verbally. The notion that a motor response has access to an independent spatial representation of the visual scene has been confirmed by investigations of Bruno and Bernardis (in press). Neither Kolb and Braun nor Morgan et al. had used a pointing response in their experiments. However, our attempt to elicit blindsight in normal observers using a pointing response was unsuccessful. 

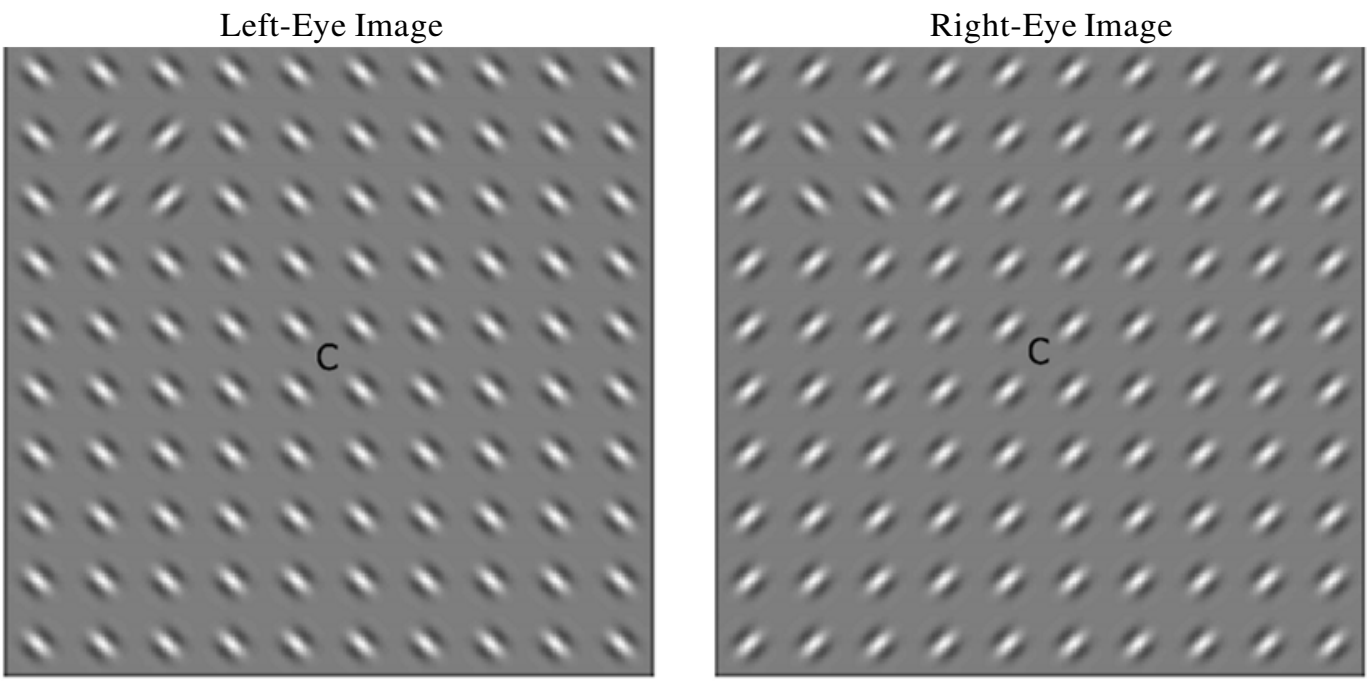

Figure 1. The stimulus complex used in the experiment. A $10 \times 10$ matrix of Gabor elements with a $2 \times 2$ target is shown for clarity, although a $20 \times 20$ matrix with a $4 \times 4$ target was actually used in the experiment. In the non rivalrous condition, the same panel was presented to both eyes. In the rivalrous condition, the orientation of Gabor elements was orthogonal across the two eyes. In the latter condition, binocular fusion of left and right images took on the appearance of a uniform plaid, eliminating any indication of the texture-defined target.

\section{METHOD}

\section{Participants}

Six participants (5 female and 1 male) with an average age of 23 years were paid $\$ 10 / \mathrm{h}$ for their participation. All had normal or corrected-to-normal visual acuity, as was assessed using a Snellen letter chart. Binocular vision was normal, as was assessed using a stereo slide viewer.

\section{Apparatus}

The stimuli were generated using a VSG2/3 Visual Stimulus Generator manufactured by Cambridge Research Systems Ltd. (Cambridge, England) and displayed on a Hitachi Superscan 21-in. monitor at a resolution of $800 \times 600$ pixels, at a frame rate of $100 \mathrm{~Hz}$. CrystalEyes stereo shutter glasses, manufactured by StereoGraphics (San Rafael, CA), were used to produce a dichoptic display, using a time-sequential method, which resulted in a display rate of $50 \mathrm{~Hz}$ per eye. The shutters had a light transmittance of $30 \%$ and response times of 0.2 and $2.8 \mathrm{msec}$ for closing and opening, respectively. Peak monitor luminance was $70 \mathrm{~cd} / \mathrm{m}^{2}\left(21 \mathrm{~cd} / \mathrm{m}^{2}\right.$ through the shutter glasses). The experiment was controlled using an IBM-compatible computer.

\section{Stimuli}

The display was the same as that used by Kolb and Braun (1995) and consisted of a $20 \times 20$ array of two-dimensional Gabor patches, each $0.5^{\circ}$ in diameter, with a period of $0.466^{\circ}$ and a standard deviation of $0.233^{\circ}$ (see Figure 1). The entire array was $10^{\circ} \times 10^{\circ}$ of visual angle at a viewing distance of $114 \mathrm{~cm}$. The target comprised an area of $4 \times 4$ patches $\left(2^{\circ} \times 2^{\circ}\right)$ at orthogonal orientation from the other patches and was centered in one of the four quadrants of the display. The target appeared with equal frequency in each of the quadrants. A $0.5^{\circ}$ Landolt $\mathrm{C}$ was situated at the center of the display, as a check on central fixation. In the nonrivalrous condition, each eye saw an identical stimulus complex. In the rivalrous condition, each eye saw a stimulus complex with patterns in complementary, orthogonal orientation. A gray screen with a central fixa- tion crosshair of $1^{\circ}$ was displayed between stimulus presentations. Ambient light provided by indirect illumination with an incandescent light bulb was held at $5 \mathrm{~cd} / \mathrm{m}^{2}$.

\section{Design}

There were two within-subjects factors in the experiment: response method (verbal vs. pointing) and display type (rivalrous vs. nonrivalrous). They were combined factorially, yielding a total of four experimental conditions.

\section{Procedure}

Each participant completed a preliminary calibration procedure, 6 practice sessions, and 12 experimental sessions. During the calibration procedure, stimulus contrast $(20 \%-40 \%)$ and display duration $(30-250 \mathrm{msec})$ were adjusted to achieve a mean accuracy level of approximately $80 \%$ for rivalrous and nonrivalrous display types. This was done with an adaptive psychophysical procedure (Taylor \& Creelman, 1967), using the verbal-response method.

Following calibration, each participant completed three practice sessions with each response method. The actual experiment consisted of 3 blocks of 60 trials in each of the four conditions, for a total of 12 blocks. Display type and response method were constant throughout a session. The order of the blocks was randomized for each participant. Each participant completed the experiment in three to four 1-h sessions.

The trials were self-paced, and each trial was initiated with a buttonpress. Following display presentation, the participants gave three responses: First, they indicated the location of the target quadrant $(1,2,3$, or 4: upper left, upper right, lower left, and lower right, respectively); second, they reported the direction of the Landolt $\mathrm{C}$ (up, down, left, or right); finally, they reported a confidence rating, on a scale of 1-10, indicating their degree of certainty about the localization response. A confidence rating of 1 indicated that they were merely guessing in making the localization response, whereas a rating of 10 indicated absolute certainty that they had correctly localized the target. The participants were urged to use the entire range of confidence ratings and to respond without reflection, but not so 
fast as to compromise accuracy. The experimenter recorded the responses on the computer. Trials on which the wrong orientation of the Landolt $\mathrm{C}$ was reported were discarded and repeated later in the block. This occurred rarely, on about $1 \%-2 \%$ of the trials.

Depending on the experimental condition (verbal vs. pointing), the participants indicated the quadrant of the display containing the target either by saying the numbers $(1,2,3$, or 4$)$ or by pointing with the index finger at the target. The direction of the Landolt $\mathrm{C}$ and the confidence rating were always reported verbally.

We stress that all aspects of the experiment that might be deemed critical for a successful replication, such as type and number of micropatterns, fixation control, size of target area, degree of practice, and response procedures in the verbal-response condition, were identical to those used by Kolb and Braun (1995).

\section{RESULTS AND DISCUSSION}

The results of the experiment are shown in Figure 2. Within each panel, the bars indicate the localization accuracy at the confidence rating shown on the $x$-axis. For convenience, Kolb and Braun's (1995) results have been replotted in the first column. The results obtained in the present work for verbal and pointing responses are shown in the second and third columns, respectively. The results for the nonrivalrous and rivalrous display types are shown in the top and bottom rows, respectively.

Consider the nonrivalrous viewing condition (top panels of Figure 2), in which Gabor patches were presented at the same orientation to each eye. The results in this condition were not controversial, and were in accord with those of Kolb and Braun (1995): Accuracy and confidence ratings were correlated, in that accuracy increased as confidence ratings increased; that is, the participants were able to judge the accuracy of their localization responses (Kendall's $\tau=.78, p<.002$ for verbal responses; Kendall's $\tau=.85, p<.001$ for pointing responses).

Consider next the results in the critical, rivalrous viewing condition (bottom panels of Figure 2), in which each eye saw a complementary image. Here, our results differed from those of Kolb and Braun (1995). Whereas they found no correlation between localization accuracy and confidence ratings, our data showed a positive correlation between these two variables (Kendall's $\tau=.72$, $p<.004$ for verbal responses; Kendall's $\tau=.87, p<.001$ for pointing responses). In our experiment, the observers were capable of assessing their level of accuracy in locating the target quadrant in the rivalrous condition, implying conscious awareness of the target's location. Without exception, all of the observers in this experiment and in pilot experiments yielded this pattern of results. Thus, we found no indication of blindsight using either the verbal- or the pointing-response method. Evidently, the pointing response did not provide access to an exclusively unconscious visuomotor representation. When interviewed after the experiment, the observers indicated that in the rivalrous condition all the micro-

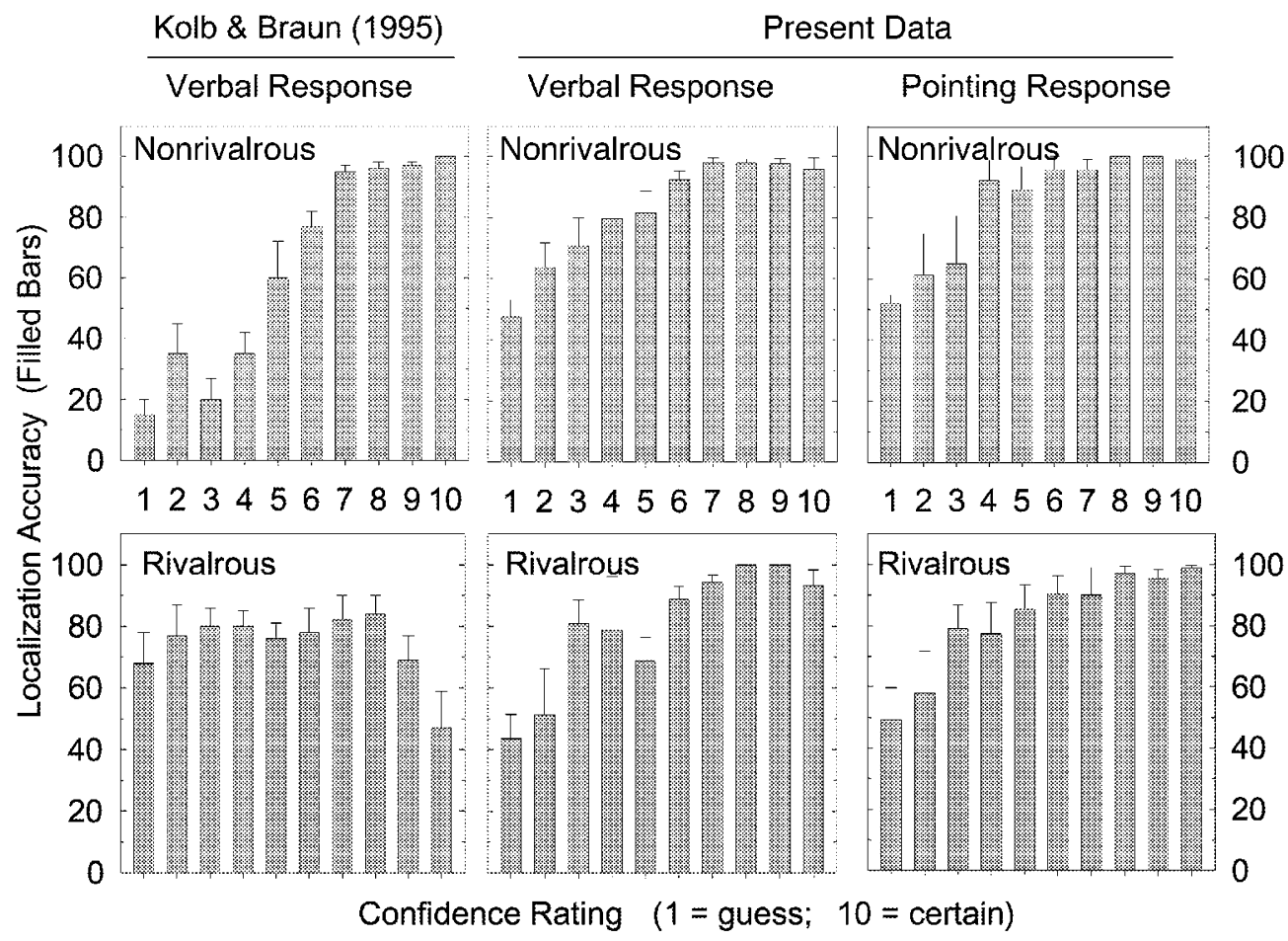

Figure 2. The left column shows the results of Kolb and Braun (1995), and the other two columns show the results of the present experiment for the verbal-response (center column) and pointing-response (right column) conditions. The results for the nonrivalrous and rivalrous displays are shown in the top and bottom rows, respectively. 
patterns appeared completely fused, yet the observers had a vague sense of the target, as if a faint boundary existed between the target area and the rest of the display, in agreement with Morgan et al. (1997).

In our report and in Kolb and Braun (1995), the overall level of localization accuracy was about $80 \%$ in the rivalrous condition. Thus, the important difference between the two studies was not in localization accuracy, but in the finding that confidence ratings and localization accuracy were correlated in the present work but were uncorrelated in Kolb and Braun.

In sensory psychophysics, it is normal for observers to tend to underestimate their accuracy near threshold levels (Green \& Swets, 1966). The present results are typical of this type of conservative response bias, as confidence levels were low when mean level of performance was near threshold level. The phenomenon demonstrated by Kolb and Braun (1995) in the rivalrous condition was distinct from a conservative response bias, because observers' confidence ratings were unrelated to accuracy.

It is perhaps commonplace that the brain is, to a great extent, modular, and that humans do not have conscious access to all its modular functions. In clinical blindsight, this modularity has manifested itself as an ability of patients to make fairly accurate hand and eye movements toward stimuli that they report not having seen. We concur with Georgeson (1997) that a display method capable of providing an analogue of clinical blindsight would be a valuable tool for examining the operation of the unconscious components of vision. Unfortunately, given our results and those of Morgan et al. (1997), it is clear that the rivalrous dichoptic display is not a reliable method of inducing blindsight in normal observers. Although our replication was unsuccessful, the work is valuable in that it provides closure to this widely publicized, though unfruitful, line of inquiry.

\section{REFERENCES}

Azzopardi, P., \& Cowey, A. (1998). Blindsight and visual awareness. Consciousness \& Cognition, 7, 292-311.

Bruno, N., \& Bernardis, P. (in press). Dissociating perception and action in Kanizsa's compression illusion. Psychonomic Bulletin \& Review.

Georgeson, M. (1997). Vision and action: You ain't seen nothin' yet .... Perception, 26, 1-6.

Green, D. M., \& Swets, J. A. (1966). Signal detection theory in psychophysics. New York: Wiley.

Kolb, F., \& BRAUn, J. (1995). Blindsight in normal observers. Nature, 377, 336-338.

Milner, D. A., \& Goodale, M. A. (1995). The visual brain in action. New York: Oxford University Press.

Morgan, M. J., Mason, A. J. S., \& Solomon, J. A. (1997). Blindsight in normal subjects? Nature, 385, 401-402.

Perenin, M. T., \& Jeannerod, M. (1978). Visual function within the hemianopic field following early cerebral hemidecortication in man. Neuropsychologia, 16, 1-13.

Taylor, M. M., \& Creelman, C. D. (1967). PEST: Efficiency estimates on probability functions. Journal of the Acoustical Society of America, 41, 782-787.

Weiskrantz, L., Warrington, E. K., Sanders, M. D., \& Marshall, J. (1974). Visual capacity in the hemianopic field following a restricted occipital ablation. Brain, 97, 709-728.

\section{NOTE}

1. The term blindsight originated from clinical literature (Weiskrantz, Warrington, Sanders, \& Marshall, 1974) and is still current (see, e.g., Azzopardi \& Cowey, 1998). It refers to the sparing of visuomotor ability in the presence of subjective blindness, following damage to the optic radiations or striate cortex.

(Manuscript received August 9, 2001; revision accepted for publication January 29, 2002.) 